\title{
An anatomical study of the bicipital aponeurosis in embalmed and fresh frozen cadavers
}

\author{
Hilal Akdemir Aktaş ${ }^{1}$ (D), Sinem Akkaşoğlu ${ }^{2}$ (D), Mine Farımaz ${ }^{3}$ (D), Mustafa Fevzi Sargon ${ }^{4}$ (D) \\ ${ }^{1}$ Department of Anatomy, Hacettepe University Faculty of Medicine, Ankara, Turkey \\ ${ }^{2}$ Department of Anatomy, Ankara Yildrrm Beyazıt University Faculty of Medicine, Ankara, Turkey \\ ${ }^{3}$ Department of Anatomy, Ufuk University Faculty of Medicine, Ankara, Turkey \\ ${ }^{4}$ Department of Anatomy, Lokman Hekim University Faculty of Medicine, Ankara, Turkey
}

\begin{abstract}
Objectives: The bicipital aponeurosis is a fascial expansion which arises from the distal tendon of biceps brachii muscle. It is an important structure for protecting the median nerve and brachial artery. The aim of this study was to analyze the morphometry and shape of the bicipital aponeurosis and its implications for the protection of the median nerve and brachial artery.

Methods: Upper extremities of two fresh frozen and seven embalmed cadavers (five right, four left sides) were dissected. The ages of the cadavers varied between 60-86 years. The central length, superior width, central width, inferior width and the shape of bicipital aponeurosis were evaluated. All measurements were performed by using digital caliper.

Results: The central length of the bicipital aponeurosis was measured $3.6 \pm 1.2 \mathrm{~cm}$. The superior, central and inferior width of the bicipital aponeurosis were found $1.5 \pm 0.7 \mathrm{~cm}, 1.5 \pm 0.6 \mathrm{~cm}$ and $1.8 \pm 0.8 \mathrm{~cm}$, respectively. Through the examination of upper extremities; two different shapes of bicipital aponeurosis were observed. In type l; the bicipital aponeurosis was fusiform in shape and observed in four upper extremities. In five extremities, it was found as quadrangular in shape and classified as type II.

Conclusion: The morphometry and shape of bicipital aponeurosis have a clinical importance to protect the median nerve and brachial artery or to reduce compression of these neurovascular structures. A better understanding of bicipital aponeurosis morphometry is important in assessment of biomechanical properties of biceps brachii.
\end{abstract}

Keywords: bicipital aponeurosis; brachial artery; median nerve; morphometry

Anatomy 2021;15(2):99-103 @2021 Turkish Society of Anatomy and Clinical Anatomy (TSACA)

\section{Introduction}

The bicipital aponeurosis is a fascial expansion which arises from the distal tendon of biceps brachii muscle. ${ }^{[1]}$ It is an important structure for the protection of the median nerve and brachial artery. ${ }^{[2]}$ There are several functions attributed to the bicipital aponeurosis in the literature. These functions are as follows: (1) Increasing the tension of biceps brachii tendon; ${ }^{[3]}(2)$ reinforcement of the ante-brachial fascia; ${ }^{[4]}(3)$ pulling the posterior border of the ulna medially to do supination of the forearm; ${ }^{[5]}$ (4) stabilization of the distal biceps brachii tendon; ${ }^{[6]}(5)$ feedback role between the fascia and biceps brachii muscle..$^{[7]}$
The aim of this study was to analyze the morphometry and shape of the bicipital aponeurosis and its implications for the protection of the median nerve and brachial artery.

\section{Materials and Methods}

In this study, upper extremities of two fresh frozen and seven embalmed cadavers ( 4 female, 5 male; five right, four left sides) were dissected. The ages of the cadavers varied between $60-86$ years. All of the upper extremities were free from pathology, trauma, surgical incision or deformity. All procedures were approved by the Ethical Committee of Lokman Hekim University and the study was conducted in the gross anatomy dissection laborato-

This study was presented as a poster at the Winter Meeting of the British Association of Clinical Anatomists, Univeristy of Leeds, 15 December 2016, Leeds, UK. 
ry of Hacettepe University. The measurements were performed by using digital caliper.

The parameters evaluated in this study were as follows: (1) The central length of the bicipital aponeurosis; (2) The superior width of the bicipital aponeurosis; (3) The central width of the bicipital aponeurosis; (4) The inferior width of the bicipital aponeurosis; (5) The shape of the bicipital aponeurosis (Figure 1).

\section{Results}

Two fresh frozen and seven embalmed upper extremities (4 female, 5 male; five right, four left sides) were dissected in this study. Through the examination of upper extremities; two different shapes of bicipital aponeurosis were observed. In type I; the bicipital aponeurosis was fusiform in shape and observed in four upper extremities (Figure 2). In these cadavers, bicipital aponeurosis run obliquely and covered the median nerve and brachial artery. In only one extremity, the bicipital aponeurosis was fusiform in shape, but membranous in structure and it was difficult to separate it from the antebrachial fascia (Figure 3).

In five of the upper extremities, it was quadrangular in shape and classified as type II (Figure 4). Quadrangular bicipital aponeurosis was wider and thicker than the fusiform shaped ones.

The mean central length of the bicipital aponeurosis (A-B) was $3.3 \pm 0.6 \mathrm{~cm}$ (min-max: $2.8-3.9 \mathrm{~cm}$ ) on the left side, $3.8 \pm 1.5 \mathrm{~cm}$ (min-max: $1.8-5.9 \mathrm{~cm}$ ) on the right side and $3.6 \pm 1.2 \mathrm{~cm}$ in general. The mean superior width $(\mathrm{C}-\mathrm{D})$ was $1.5 \pm 0.4 \mathrm{~cm}$ (min-max: $1.3-2.2 \mathrm{~cm}$ ) on the left side, $1.5 \pm 0.7 \mathrm{~cm}$ (min-max: $0.7-2.8 \mathrm{~cm}$ ) on the right side and $1.5 \pm 0.6 \mathrm{~cm}$ in general. The central width (E-F) was found to be $1.4 \pm 0.3 \mathrm{~cm}$ (min-max: $1.1-1.7 \mathrm{~cm}$ ) on the left side, $1.6 \pm 0.9 \mathrm{~cm}$ (min-max: $0.7-3.1 \mathrm{~cm})$ on the right side and $1.5 \pm 0.6 \mathrm{~cm}$ in general. The inferior width of the bicipital aponeurosis $(\mathrm{G}-\mathrm{H})$ was $1.7 \pm 0.4 \mathrm{~cm}(\min -\max : 1.3-2.1 \mathrm{~cm})$ on the left side, $1.9 \pm 1.0 \mathrm{~cm}$ (min-max: $0.8-3.5 \mathrm{~cm}$ ) on the right side and $1.8 \pm 0.8 \mathrm{~cm}$ in general. There was no statisti-
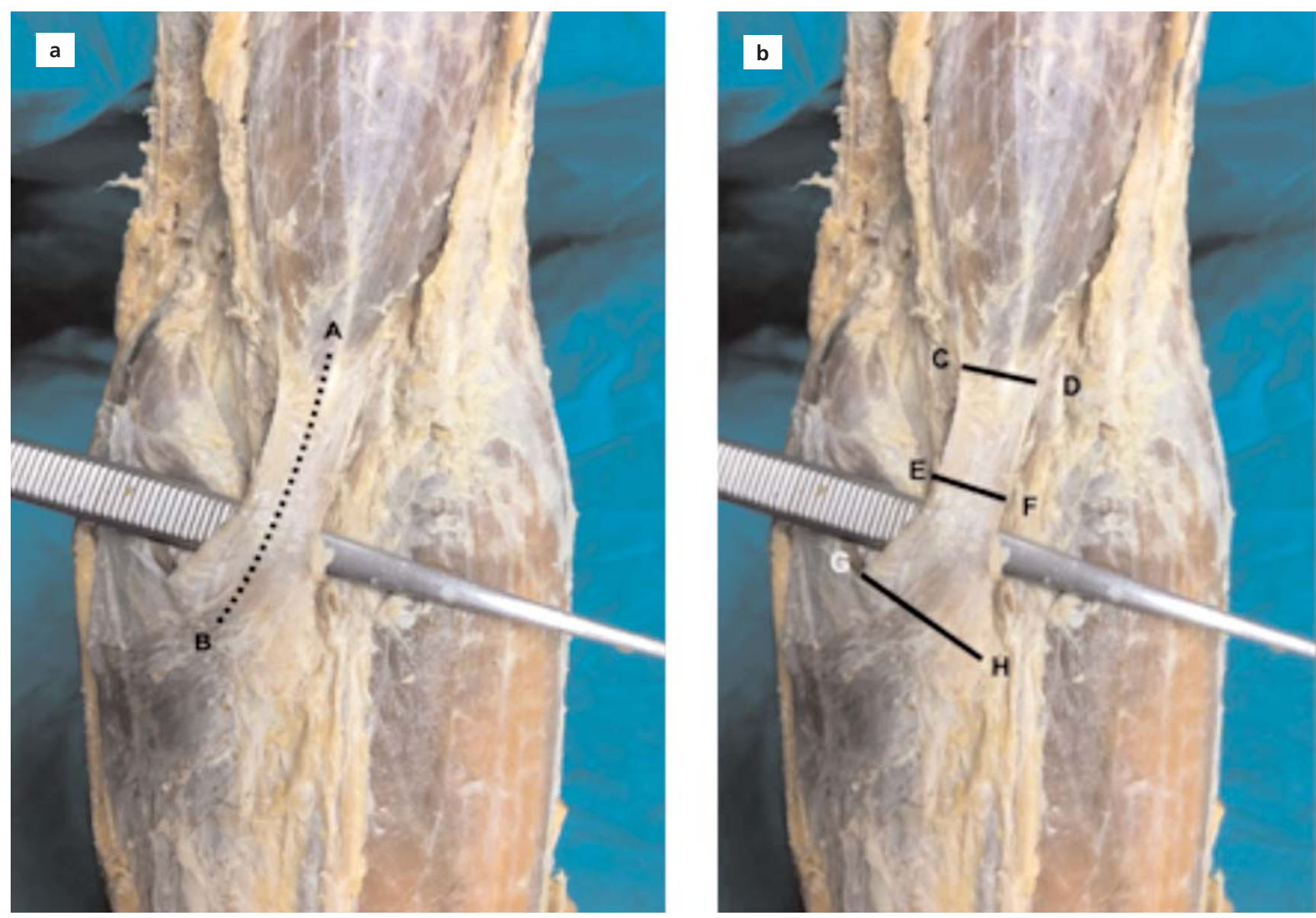

Figure 1. Figures (a) and (b) show the parameters measured in this study. A-B: the central length of the bicipital aponeurosis; C-D: the superior width of the bicipital aponeurosis; E-F: the central width of the bicipital aponeurosis; G-H: the inferior width of the bicipital aponeurosis. 


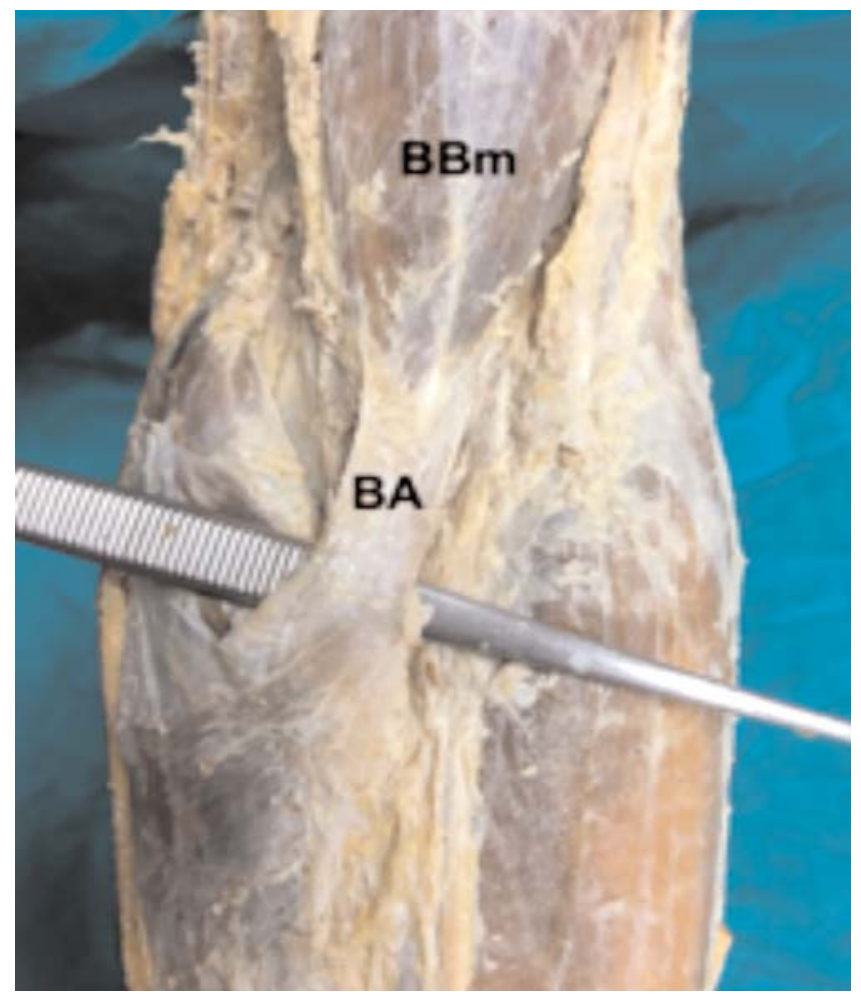

Figure 2. Fusiform bicipital aponeurosis (Type I). BA: bicipital aponeurosis; $\mathrm{BBm}$ : biceps brachii muscle.

cally significant relationship between the variables and among right and left sides $(\mathrm{p}>0.05)$.

\section{Discussion}

The bicipital aponeurosis was described as a fibrous lamina arising from the medial margin of the distal biceps brachii tendon. It runs distally and medially, broadens, and joins with the antebrachial fascia. It surrounds the flexor muscles of forearm as well as the brachial artery and median nerve. ${ }^{[7,8]}$ The flexor-pronator muscles can be compressed by the bicipital aponeurosis during pronation against resistance. ${ }^{[8]}$ It can cause compression of the median nerve and brachial artery, by narrowing the space under it. ${ }^{[8,9]}$

Snoeck et al. ${ }^{[7]}$ dissected 50 upper extremities of 36 embalmed cadavers (23 females, 13 males) and measured the central length of the bicipital aponeurosis as $8.6 \pm 1.0$ $\mathrm{cm}$. Caetano et al. ${ }^{[0]}$ dissected 60 upper limbs of 30 cadavers (26 males, 4 females) and found that the length of aponeurosis ranged from 4.5 to $6.2 \mathrm{~cm}$. In this study, the central length was found to be $3.6 \pm 1.2 \mathrm{~cm}$, which was lower than the previous studies in the literature.

Caetano et al. ${ }^{[9]}$ reported that the width of the bicipital aponeurosis ranged from 0.5 to $2.6 \mathrm{~cm}$. Snoeck et al. ${ }^{[7]}$ measured the mean width as $0.9 \pm 0.4 \mathrm{~cm}$ superiorly,

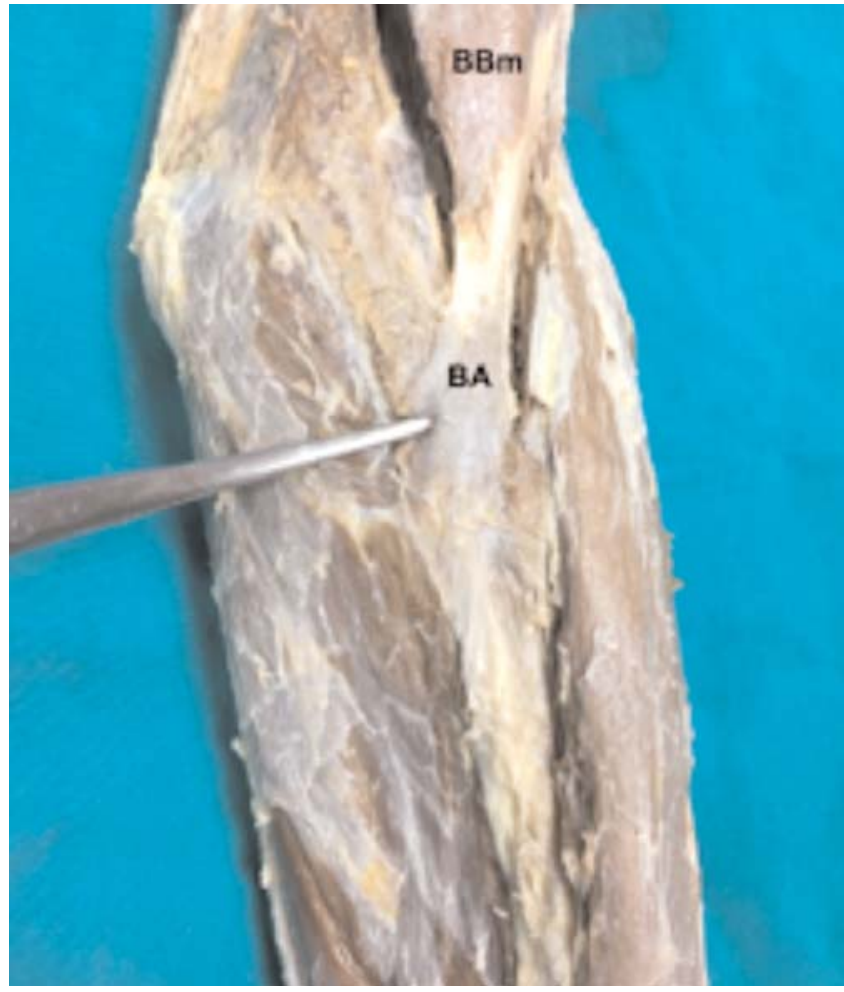

Figure 3. Fusiform bicipital aponeurosis in membranous structure. BA: bicipital aponeurosis; BBm: biceps brachii muscle.

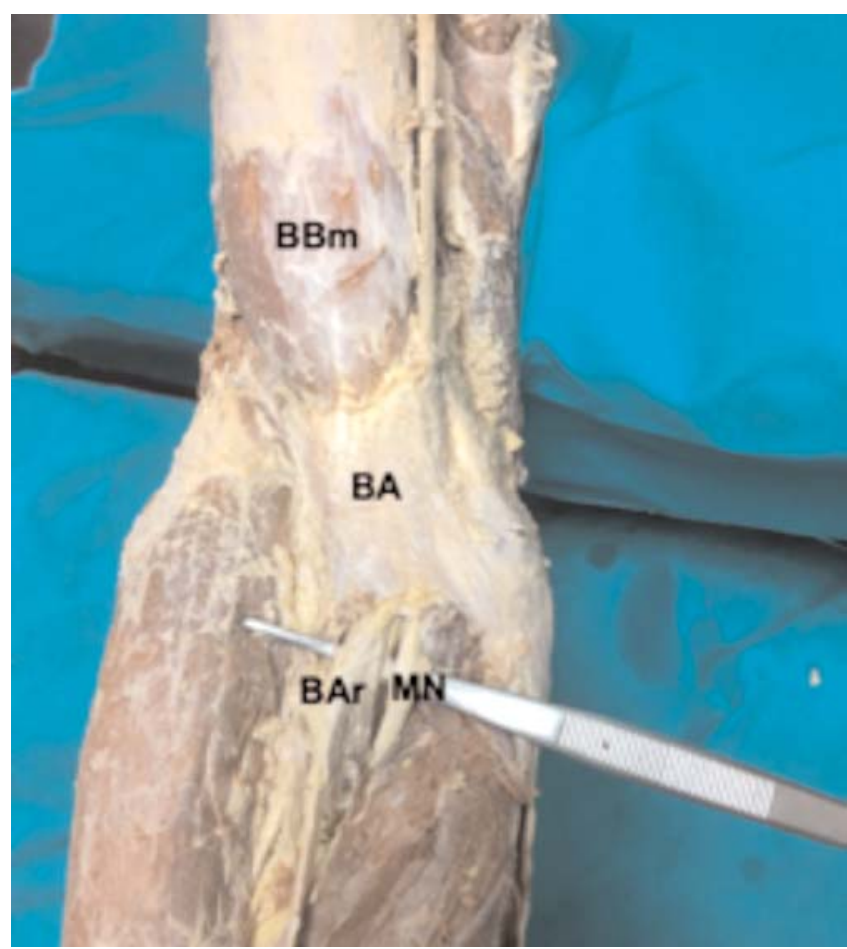

Figure 4. Quadrangular bicipital aponeurosis (Type II). BA: bicipital aponeurosis; BAr: brachial artery; BBm: biceps brachii muscle; $\mathrm{MN}$ : median nerve. 
$1.2 \pm 0.4 \mathrm{~cm}$ centrally and $1.0 \pm 0.4 \mathrm{~cm}$ inferiorly. Joshi et al. ${ }^{[10]}$ dissected 30 cadaveric upper limbs (16 right, 14 left side) and measured the mean width of bicipital aponeurosis $15.74 \mathrm{~mm}$ on the right and $17.57 \mathrm{~mm}$ on the left side. In this study, the mean superior, central and inferior width of the bicipital aponeurosis were found to be $1.5 \pm 0.7 \mathrm{~cm}$, $1.5 \pm 0.6 \mathrm{~cm}$ and $1.8 \pm 0.8 \mathrm{~cm}$, respectively. Our results were compatible with the other studies in the literature. The width of the bicipital aponeurosis is important clinically because hypertrophy or enlargement of the aponeurosis may cause compression of the median nerve. ${ }^{[9,11]}$ Caetano et al. ${ }^{[9]}$ observed that the bicipital aponeurosis which was very narrow and thin, was unlikely to compress the median nerve. Martinelli et al. ${ }^{[1]}$ reported one case with median nerve compression caused by a thick and well-developed bicipital aponeurosis. The knowledge of normal dimensions of the aponeurosis will help the physicians to diagnose nerve compression syndromes accurately.

Caetano et al. ${ }^{[9]}$ showed that it had a rectangular formation in most cases, while it was trapezoidal in few cases. We observed two different morphological shapes of bicipital aponeurosis as follows: quadrangular shape in five cases and fusiform shape in four cases. Similar to Caetano et al., ${ }^{[9]}$ quadrangular shape was frequently detected in present study. We suggest that quadrangular and fusiform shapes were effective for the protection of the brachial artery and median nerve, except the bicipital aponeurosis in membranous structure. However, since quadrangular shaped aponeurosis surrounds the neurovascular structures tightly, it may be a risk factor for the compression of the neurovascular structures.

Caetano et al. ${ }^{[9]}$ demonstrated that the short and long heads of the biceps brachii muscle contributed to the formation of the bicipital aponeurosis in 55 limbs, and the most significant contribution was always from the short head. In three limbs, only the short head contributed to the formation of the bicipital aponeurosis. Joshi et al. ${ }^{[10]}$ reported that the fibers from the short head formed the proximal part of aponeurosis, while the long head contributed to the distal part of bicipital aponeurosis. Athwal et al. ${ }^{[12]}$ dissected 15 fresh-frozen upper extremities and showed that the aponeurosis originated from the short head of biceps in all specimens. Dirim et al. ${ }^{[5]}$ made a dissection of 17 upper limbs (7 left, 10 right side; 9 male, 8 female) and they found that the bicipital aponeurosis was formed by superficial tendinous fibers arising from both muscle bellies in most cases (94.1\%). In 5.9\% (1/17) of the specimens, only fibers of the short head contributed to the bicipital aponeurosis. The structures that contribute to the formation of bicipital aponeurosis are still controversial in the literature. Further investigation with a larger samples is required to better understand bicipital aponeurosis formation.
Repairing the bicipital aponeurosis in cases with distal biceps tendon rupure is controversial in clinical practice. Some researchers reported that the presence of an intact bicipital aponeurosis limited retraction of the distal biceps tendon and increased the chance of direct repair regardless the time of the injury. ${ }^{[13,14]}$ Landa et al. ${ }^{[3]}$ performed a cadaveric study and showed that termino-terminal sutures of the bicipital aponeurosis increased the mechanical strength in distal biceps tendon repair. Conlin et al. ${ }^{[15]}$ treated 24 male patients with a distal biceps tendon rupture. They reported that repair of the bicipital aponeurosis in conjunction with distal biceps tendon repair led to a faster return to activities compared with isolated tendon repair. Fontana et al. $^{[16]}$ described a new surgical technique as treating distal biceps tendon tear with bicipital aponeurosis augmentation. They used autologous vascularized bicipital aponeurosis graft and found that recovery time was quicker and integration was faster than the techniques described in the literature. The topographic anatomy of the bicipital aponeurosis is essential for the successful repair of the aponeurosis and distal biceps tendon.

\section{Conclusion}

In conclusion; the morphometry and shape of bicipital aponeurosis have a clinical importance to protect the median nerve and brachial artery or to reduce compression of these neurovascular structures. A better understanding of bicipital aponeurosis morphometry is a great value in assessment of biceps brachii movement biomechanics.

\section{Acknowledgments}

We would like to thank the cadaver donors without whom such research would not be possible.

\section{Conflict of Interest}

The authors declare that they have no conflict of interest.

\section{Author Contributions}

HAA: project development, data collection, manuscript writing; SA: project development, data analysis, manuscript editing; MF: project development, data analysis, manuscript writing and editing; MFS: data analysis, statistical evaluation, manuscript editing.

\section{Ethics Approval}

All procedures were approved by the Ethical Committee of Lokman Hekim University (approval date: 09.08.2021, approval number: 2021/088).

\section{Funding}

None. 


\section{References}

1. Congdon ED, Fish HS. The chief insertion of the bicipital aponeurosis is on the ulna; a study of collagenous bundle patterns of antebrachial fascia and bicipital aponeurosis. Anat Rec 1953;116:395407.

2. Eames MHA, Bain GI, Fogg QA, van Riet RP. Distal biceps tendon anatomy: a cadaveric study. J Bone Joint Surg Am 2007;89:1044-9.

3. Landa J, Bhandari S, Strauss EJ, Walker PS, Meislin RJ. The effect of repair of the lacertus fibrosus on distal biceps tendon repairs: a biomechanical, functional, and anatomic study. Am J Sports Med 2009;37:120-3.

4. Blasi M, de la Fuente J, Martinoli C, Blasi J, Perez-Bellmunt A, Domingo T, Miguel-Perez M. Multidisciplinary approach to the persistent double distal tendon of the biceps brachii. Surg Radiol Anat 2014;36:17-24.

5. Dirim B, Brouha SS, Pretterklieber ML, Wolff KS, Frank A, Pathria $\mathrm{MN}$, Chung CB. Terminal bifurcation of the biceps brachii muscle and tendon: anatomic considerations and clinical implications. AJR Am J Roentgenol 2008;191:248-55.

6. Vanhees M, van Riet RP. Reconstruction after distal biceps tendon rupture. Journal of Orthopaedics, Trauma and Rehabilitation 2012; $16: 2-8$.

7. Snoeck O, Lefevre P, Sprio E, Beslay R, Feipel V, Rooze M, Van Sint Jan S. The lacertus fibrosus of the biceps brachii muscle: an anatomical study. Surg Radiol Anat 2014;36:713-9.

ORCID ID:

H. Akdemir Aktaş 0000-0002-7353-8069; S. Akkașoğlu 0000-0002-3371-4734; M Farımaz 0000-0002-9621-1616: M. Fevzi Sargon 0000-0001-6360-6008
8. Bassett FHI, Spinner RJ, Schroeter TA. Brachial artery compression by the lacertus fibrosus. Clin Orthop Relat Res 1994;(307):110-6.

9. Caetano EB, Vieira LA, Almeida TA, Gonzales LAM, Bona JE, Simonatto TM. Bicipital aponeurosis. Anatomical study and clinical implications. Rev Bras Ortop 2018;53:75-81.

10. Joshi SD, Yogesh AS, Mittal PS, Joshi SS. Morphology of the bicipital aponeurosis: a cadaveric study. Folia Morphol (Warsz) 2014;73: 79-83.

11. Martinelli P, Gabellini AS, Poppi M, Gallassi R, Pozzati E. Pronator syndrome due to thickened bicipital aponeurosis. J Neurol Neurosurg Psychiatry 1982;45:181-2.

12. Athwal GS, Steinmann SP, Rispoli DM. The distal biceps tendon: footprint and relevant clinical anatomy. J Hand Surg Am 2007;32: 1225-9.

13. O'Driscoll SW, Goncalves LB, Dietz P. The hook test for distal biceps tendon avulsion. Am J Sports Med 2007;35:1865-9.

14. Bosman HA, Fincher M, Saw N. Anatomic direct repair of chronic distal biceps brachii tendon rupture without interposition graft. J Shoulder Elbow Surg 2012;21:1342-7.

15. Conlin CE, Naderipour A, ElMaraghy A. Outcome of distal biceps tendon repair with and without concomitant bicipital aponeurosis repair. Orthop J Sports Med 2019;7:2325967119865500.

16. Fontana M, Trimarchi A, Colozza A. Lacertus fibrosus augmentation for distal biceps brachii rupture repair: surgical technique. Musculoskelet Surg 2016;100:85-8.

Correspondence to: Hilal Akdemir Aktaş, MD, PhD

Department of Anatomy, Hacettepe University Faculty of Medicine,

06100, Ankara, Turkey

Phone: +903123052109

e-mail: hakdemir06@hotmail.com

Conflict of interest statement: No conflicts declared.

This is an open access article distributed under the terms of the Creative Commons Attribution-NonCommercial-NoDerivs 4.0 Unported (CC BY-NCND4.0) Licence (http://creativecommons.org/licenses/by-nc-nd/4.0/) which permits unrestricted noncommercial use, distribution, and reproduction in any medium, provided the original work is properly cited. How to cite this article: Akdemir Aktaş H, Akkaşoğlu S, Farımaz M, Sargon MF. An anatomical study of the bicipital aponeurosis in embalmed and fresh frozen cadavers. Anatomy 2021;15(2):99-103. 